
LA-UR-
$91-3825$
10382
DE92 003791

$L A-U R--91-3825$

Los Alamos National Laboation is operated by the University of California for the United States Department of Energy under contract W-7405 ENG-36

\title{
TITLE: THE LOS ALAMOS FEL PHOTOINJECTOR DRIVE LASER
}

\author{
AUTHOR(S): James Early, CLS-6 \\ Jerome Barton, CLS-6 \\ George Busch, CLS -6 \\ Robert Wenzel, CLS-6 \\ Dennis Remelius, CLS-6
}

\section{SUBMITTED TO: Proceedings for Poster presentation for the Thirteenth International Free-Electron Laser Conference, August 25-30, 1991, Santa Fe, NM}

\begin{abstract}
By acceptance of this article, the publisher recognizes that the U. S. Government retains a nonexclusive, royalty-free license 10 publish or reproduce the published form of this contribution or to allow others to do so, for Government purposes.

The Los Alamos National Laboratory requests that the publisher identify this article as work pertormed under the auspices of the U. S. Department of Energy.
\end{abstract}

\section{$\measuredangle\left(\mathrm{O} S \mathrm{~A} \cap\left(\mathrm{O} S \begin{array}{l}\text { Los Alamos National Laboratory } \\ \text { Los Alamos, New Mexico } 87545\end{array}\right.\right.$ DISCLAIMER}

This report was prepared as an account of work sponsored by an agency of the United States Government. Neither the United States Government nor any agency thereof, nor any of their employees, makes any warranty, express or implied, or assumes any legal liability or responsibility for the accuracy, completeness, or usefulness of any information, apparatus, product, or process disclosed, or represents that its use would not infringe privately owned rights. Reference herein to any specific commercial product, process, or service by trade name, trademark, manufacturer, or otherwise does not necessarily constitute or imply its endorsement, recommendation, or favoring by the United States Government or any agency thereof. The views and opinions of authors expressed herein do not necessarily state or reflect those of the United States Government or any agency thereof.

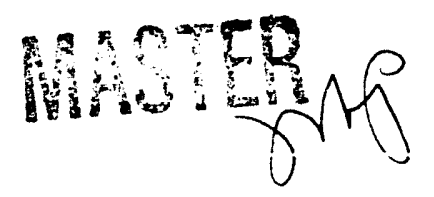


The Los Alamos FEL Photo-Injector Drive Laser •

\author{
J.Early, J.Barton, G.Busch, R.Wenzel and D.Remelius \\ Chemical and Laser Sciences Division \\ Los Alamos National Laboratory \\ Los Alamos, New Mexico 87545
}

\begin{abstract}
The drive laser system of the photoelectric injector for the Los Alamos free - electron laser (FEL) is described. The bright electron beams required for high performance FELs demand stringent specifications on drive laser output levels, beam quality, and stability. The laser system generates 7-15 ps ( FWHM ) Gaussian pulses of $527 \mathrm{~nm}$ light at a repetition rate of $21.67 \mathrm{MHz}$. These "micropulses" are contained within a $<200$ microsec long "macropulse" envelope which is emitted from the drive laser at a $1 \mathrm{~Hz}$ rate. Micropulse energies as large as 12 microjoules are obtained. The design specifications, laser architecture, and operating performance following the most recent round of upgrades are presented.
\end{abstract}

\title{
I) Introduction
}

The Los Alamos FEL incorporates a high-brightness, lowemittance electron source known as the photoelectric injector ( refs 1 and 2 describe early Nd:YAG based photo-injector development studies at Los Alamos). This device consists basically of a high current density ( $>500$ ampere $\left./ \mathrm{cm}^{2}\right)$ photo-emitter and its source of illumination - the drive laser. The pulsed drive laser produces short, picosecond duration pulses of visible light at high peak power. Each pulse of light impinging upon the photoemitter induces a prompt emission of electrons permitting very short (7-15 ps), high intensity electron pulses ( 5-10 nC) to be generated $[3-6]$.

* Work supported and funded by the US Department of Defense, Army Strategic Defense Command, under the auspices of the US Department of Energy. 
The drive laser design and operating specifications conform to the low duty-cycle operating format of the Los Alamos FEL, which requires light pulses to be applied to the photo-emitter electron source for a small fraction $(\leq .02 \%)$ of the FEL's $1 \mathrm{~Hz}$ cycle period. The drive laser output temporal format must be characterized in two different time domains: the micropulse ( a pulse of visible light lasting a few picoseconds) and the macropulse ( an envelope containing from one to $\sim 4000$ micropulses , repeated at a $21.67 \mathrm{MHz}$ rate). The macropulse is repeated at a $1 \mathrm{~Hz}$ frequency. The low duty-cycle requirement of the photoinjector permits the adoption of a pulsed drive laser light pump source which permits thermal loading in laser amplifiers to remain low. The operating characteristics of the present Los Alamos drive laser are summarized in Table 1.

\section{III) Drive Laser Architecture}

The drive system, outlined in Fig 1, consists primarily of four components; a mode-locked oscillator lasing at a wavelength of 1.053 micron, a pulse compressor, two amplifier stages, and a frequency doubler to obtain visible light in the drive laser output. In addition, two electro-optic shutters (EOS) are used one provides pulse repetition frequency reduction, while the other EOS is used to form the macropulse.To prevent mode-locked oscillator instabilities due to scattered or reflected light feedback, Faraday rotator optical isolators are used at several critical locations within the drive laser. since a detailed description of drive laser architecture is given in ref [7], a brief overview of the drive laser configuration is provided below.

\section{a) The Oscillator}

A commercially available neodymium:yttrium lithium fluoride ( Nd:YLF) mode-locked laser ( a specially modified Quantronix Model 496 ) was selected to best meet the specifications of Table 1. Factors leading to the selection of YLF over YAG as the Nd host material are discussed in ref [7]. The oscillator is operated on the pi-polarized transition at 1.053 micron with a pulse repetition rate of $108.33 \mathrm{MHz}$ ( the $12^{\mathrm{t}}$ sub-harmonic of the FEL linac's master oscillator). The $1.35 \mathrm{~nm}$ bandwidth of $\mathrm{Nd}$ : YLF supports the generation of $30-40$ ps wide pulses directly out of the oscillator.

Critical drive laser-linac synchronization is achieved by driving the acousto-optic mode-locker with a $54.17 \mathrm{MHz}$ signal derived from the rf linac's master oscillator. To more precisely control critical drive laser timing, a commercially available active phase stabilizer (Lightwave Series 1000 ) is incorporated within the oscillator. The latter reduces timing jitter relative to the rf phase from -10 ps to $<2$ ps. The oscillator mode- 
locked CW output power is typically about $6.0 \mathrm{~W}$. An amplitude stabilizer ( Quantronix Model 366 ) is used to reduce oscillator ripple to $<1.0 \%$.

\section{b) Pulse Compression}

Since the temporal widths of the mode-locked pulses directly out of the oscillator are too broad to generate a useful, low energy spread electron beam within the FEL linac, further reduction in pulse width is required. This is accomplished by using an optical fiber - multipass grating pulse compressor, see fig 2. The mode-locked light is first chirped ( linearly swept in frequency, within the 5.0 micron diameter, 2.5-m long fiber and then temporally compressed via the frequency dispersive delayline of the multi-pass grating. Using this technique, the light pulses are reduced in width from 30 ps to 7 ps, giving a compression ratio of $-4: 1$. The temporal profile of a typical compressed pulse is shown in Fig 3. Pulse compressor transmission is typically 25-30 $\%$, in which light injection into the fiber accounts for about half the total loss.

\section{c) Pulse Repetition Frequency Reduction}

In order for drive laser light to illuminate the photoemitter electron source at a $21.67 \mathrm{MHz}$ rate, as required to match the FEL resonator cavity round-trip time, a five-fold reduction in mode-locked pulse repetition frequency must be performed. This is accomplished using a fast ammonium di-deuterium phosphate ( $A D * P$ ) Pockels cell electro-optic shutter system, which selectively transmits every fifth $108.33 \mathrm{MHz}$ light pulse. Rejected micropulses are attenuated by a factor of $150: 1$.

\section{d) Amplifier System}

The pulsed amplifier system consists of two Nd:YLF amplifiers, each of which is xenon flashlamp pumped. Each amplifier rod is $100 \mathrm{~mm}$ long and $4 \mathrm{~mm}$ in diameter. The first of these amplifiers is double-passed, while the second is single-passed. A long beam waist is used to obtain a uniform amplifier aperture fillfactor ( $e^{-2}$ beam diameter to rod diameter) of 0.55 .

The excitation of each amplifier is provided by an eight stage pulse forming network (PFN) supplying approximately 250 joules of energy to each lamp. The PFN is designed to provide a temporally long, flat amplifier pump profile ( see fig 4 ). The amplifiers are operated under a quasi-steady state condition in which energy is extracted from the amplifier chain at a rate equal to the pump rate, as described in ref [7]. In this manner, 
each light pulse injected into the amplifier chain acquires an identical energy gain.

An amplifier total gain of approximately 7000 is obtained, providing pulse energies of 30 - 40 microjoules. An example of the amplifier output detected by a slow $s i$ photodiode, is given in Fig 5.

\section{e) Macropulse Formation}

$A$ second $A D \star P$ Pockels cell electro-optic shutter is used to select a portion of the amplified $21.67 \mathrm{MHz}$ pulse train. The selected waveform is known as the macropulse. The switching action of this shutter is timed so as to select a portion of the amplified pulse train which has uniform energy per pulse. The length of the macropulse is programmable from one to about 4000 micropulses (temporally - 40 ns to 200 microsecs in length), depending on the desired mode of FEL operation. The formation of a typical 20 microsec wide macropulse, monitored by a slow Si photodiode at the output of the Pockels cell, is demonstrated in Fig 6 .

\section{f) Visible Light Generation}

Frequency doubling to a wavelength of $527 \mathrm{~nm}$ at which the photocathode is relatively efficient, is accomplished using a potassium titanyl phosphate ( KTP) crystal. The KTP crystal currently used has the dimensions of $3 \times 3 \times 10 \mathrm{~mm}$ and is specifically cut for doubling the $1053 \mathrm{~nm}$ radiation of Nd:YLF. The entry and exit faces of the doubling crystal are anti-reflection coated. Since KTP has a large temperature bandwidth ( 25 deg C ) for the type II interaction employed, no thermostating of the doubling crystal is performed.

KTP has a large non-linear susceptibility and exhibits a high infrared to green conversion efficiency. The dependence of KTP conversion efficiency on 1.053 micron peak pulse power is shown in Fig 7. Infra-red light incident on the KTP crystal is focused to a waist diameter of about $1.0 \mathrm{~mm}$ with an angular divergence of about $0.5 \mathrm{mrad}$. For a typical 7 ps wide IR micropulse with an energy of 30 microjoules, about 10 microjoules are obtained in the green, providing a conversion efficiency of about 33 \%.

After doubling, a dielectric band-pass filter is used to remove better than $95 \%$ of the residual IR light from the doubling crystal's output. This was required to prevent imaging of the residual IR light onto the CCD camera which monitors the mode quality of the drive laser's $527 \mathrm{~nm}$ output. 


\section{g) Drive Laser Diagnostics}

The upgraded drive laser incorporates the extensive use of CCD caineras to monitor spatial beam profiles and beam alignment to provide a quick and accurate method for the detection and identification of operational aberrations. These small, $2 / 3$ inch format cameras are placed at various critical locations, as shown in Fig 8. Most of the cameras indicated are placed behind highreflection turning mirrors and image the low intensity mirror leakage. These cameras provide a $6.6 \mathrm{~mm} \times 8.8 \mathrm{~mm}$ active area and a typical signal to noise ratio of $50 \mathrm{~dB}$.

The video output of each CCD camera is directed to a dedicated video monitor for display of the beam image. Freeze frame video storage units are used to capture images which are transient in nature, such as the drive laser output mode. Video storage is updated with each drive laser cycle. Each monitor screen is fitted with a cross-hair graticle onto which the beam image is centered. By observing the beam position relative to the graticle at each monitor, the laser operator can quickly and easily identify system mis-alignments and make required corrections. Typically, monitors are positioned near the corresponding camera to allow personnel to view the video image as adjustments are being made.

Spatial beam profiles are generated by computerized intensity cross-section analysis of the digitized CCD video images. This IBM 386 computer based system, initially developed to provide electron beam diagnostics for the FEL's linac, is now being applied to the drive laser diagnostics as well. The output of all drive laser $C C D$ cameras are interfaced to the profile analysis system via a video multiplexer. Profile analysis of the CCD images has proved very useful in diagnosing variations in mode quality at various locations within the drive laser.

In addition to the CCD cameras, numerous photodetectors are distributed at critical drive laser sites where the continuous or occasional monitoring of light pulse amplitudes or pulse formats is needed. The output of oscilloscopes is typically monitored via CCD camera readout. Macropulse energy is measured before and after frequency doubling using removable high reflecting turning mirrors to intercept and re-direct the laser light into fixed energy monitors. Pulse width is monitored via autocorrelator and phase jitter is checked occasiorally with a streak camera.

For a complex optical system such as the drive laser, a considerable savings of time in both system start-up and routine maintenance is realized by using extensive diagnostics. Recent operating experience has shown a $50 \%$ reduction in system start- 
up time (typically $45 \mathrm{~min}$ ) compared to that required before the latest drive laser diagnostics upgrade.

\section{IV) Current Drive Laser Performance}

\section{a) Micropulse Energy}

The drive laser provides a typical output micropulse energy at $527 \mathrm{~nm}$ of 10 microjoules. This is a factor of eight higher than the original design specification of 1.2 microjoules. The higher micropulse energy permits the generation of the nominal charge of 5.0 nC to be produced with a photocathode quantum efficiency as low as $0.2 \%$. This capability proved valuable in permitting FEL operation to be extended by several hours under unusual conditions in which the photocathode $Q$ degraded rapidly. As a result of the higher obtainable micropulse energy, we expect to demonstrate a substantial increase in the useful photocathode lifetime from $\sim 8$ hours to several days.

The pulse-to-pulse uniformity of micropulse energy within a drive laser macropulse is determined largely by the amplitude stability of the laser oscillator. To minimize power fluctuations in the oscillator's output, an active amplitude stabilizer is employed. The stabilizer counteracts any variations in laser output power by adjusting the oscillator lamp's current in response to an error signal derived from the output of a photodiode monitoring the oscillator's output power. By employing amplitude stabilization of the oscillator, the AC amplitude ripple is reduced from $2-3$ of to $<1.0 \%$. This is demonstrated in Fig 9. The bandwidth of the stabilizer is relatively low, so only noise frequency components below $2 \mathrm{KHz}$ are effectively reduced. Droop during the drive laser macropulse as well as variation from macropulse to macropulse are each maintained at 1.0 or less.

\section{b) Drive Laser Spatial Mode Quality}

The spatial mode of the drive laser output is highly Gaussian, as demonstrated in Fig 10, where a digitized single line CCD cross-section of the laser mode is fit to a Gaussian. A slight amount of astigmatism $(<10 \%)$ is present in the mode, although this presents no significance for photoinjector performance. By preventing an over-fill of drive laser apertures, hot spots and voids within the output due to diffraction fringing are avoided. Also, the appropriate placement of cameras at critical drive laser locations permits the maintenance of precise optical alignments, thus avoiding possible aperture vignetting.

The spatial properties of the electron beam generated at the FEL's photoinjector and accelerated within the linac are depen- 
dent upon the spatial characteristics of the drive laser light illuminating the photocathode. This is aptly demonstrated in fig 11, where the drive laser light, imaged upon the photocathode to form the letters "FEL", is accurately reproduced in the accelerated electron beam profile. In this manner, variations the drive laser output mode and positioning of the laser light at the photocathode are also reflected in the stability of the generated electron beam. Drive laser light pointing stability is of particular concern with regard to the long $(-30 \mathrm{~m})$ distance over which the collimated laser light must be transported to the photocathode. Approximately $3 / 4$ of the total transport distance is in vacuum.

To determine the pointing and mode stability of the drive laser output, a CCD camera was used to monitor the variations in the laser mode's waist and position after traversing a substantial distance through air. The laser light was initially collimated to a $e^{-2}$ waist diameter of $8 \mathrm{~mm}$, and then propagated through a distance of $15 \mathrm{~m}$ to the camera, where a short focal length lens was used to reduce the beam waist to $-2.5 \mathrm{~mm}$. Digitized CCD images of the laser mode were recorded every 30 secs over a period of $12 \mathrm{~min}$. The horizontal position of the laser mode's apex (the maximum in the mode's intensity distribution) as well as the $e^{-2}$ waist diameter along the horizontal direction were determined for each CCD image. Variations in the mode position and waist relative to their mean values were calculated and expressed in a normalized fashion as a percentage of the mean beam waist. The results of these pointirig and mode stability measurements are summarized in the plots of figures 12 and 13 . The short term variations in drive laser beam waist and pointing are respectively $1.8 \%$ and $1.0 \%$ (rms) of the mean beam waist. These rms values are small and are comparable with the uncertainty in a single measurement for either of these two quantities (2.0 $\%$ and $1.0 \%$ respectively). Mode waist and position variations of this magnitude have a negligible effect on electron beam quality.

\section{c) Drive Laser Timing stability}

Timing jitter in the drive laser output causes a shift [8], as well as a broadening [9] in the FEL's accelerated electron beam energy. Although the drive laser is synchronized to the FEL's rf linac, significant shifts in relative phasing can still occur due primarily to small length variations in the oscillator cavity caused by vibrations and temperature fluctuations. To minimize drive laser timing jitter, the mode-locked oscillator has been actively phase stabilized. This is accomplished by employing a negative feedback control loop, which first detects the phase difference between the oscillator output and the modelocker's drive signal and then applies a counteracting phase 
shift to the mode-locker's rf drive signal. The bandwidth of the timing stabilizer is approximately $20 \mathrm{KHz}$.

Active phase stabilization achieves significant improvements in drive laser phase stability, as seen in Figs 14 and 15 . streak camera measurements taken over short ( $1 \mathrm{~min}$ ) time intervals, show reductions in rms phase jitter from $\sim 11$ ps without stabilization to < 1.7 ps with stabilization [8]. This latter measured value for stabilized jitter is at the measurement limit of the streak camera. The actual jitter may in fact be appreciably less.

The measured long-term phase jitter is significantly greater than short-term jitter ( see Fig 15). A monotonic drift in phase the order of $~ 20$ ps was observed over a one hour sampling period [8]. The cause of long-term phase drift is currently not understood, but may be due to slow temperature changes in the modelocked oscillator. Slowly varying phase poses no immediate problem to drive laser operation since manual phase corrections can easily be made.

Streak camera measurements of intramacropulse timing slew were also made for a $100 \mathrm{microsec}$ long macropulse. Without timing stabilization, a value of 5 ps was obtained. The corresponding value with stabilization was $<2$ ps [10].

\section{v) Conclusion}

The drive laser developmental efforts conducted at Los Alamos over the past several years have been highly successful in meeting the stringent operating demands placed on high current FEL photo-injector electron sources. As FEL development progresses, the drive laser will continue to evolve to satisfy the ever more demanding operating requirements.

\section{Acknowledgement}

We wish to thank A.H. Lumpkin for his invaluable contributions in performing the streak camera measurements of drive laser timing stability. We also would like to express our appreciation to R. Feldman for her assistance in providing the beam profile diagnostic measurements. 
References

1) J.S. Fraser and R.L. Sheffield, "High-Brightness Injectors for R.F. Driven Free-Electron Lasers," IEEE J. of Quant. Electr., vol QE-23, 1489-1496, September, 1987.

2) R.L. Sheffield, E.R. Gray, and J.S. Fraser, "The Los Alamos Photo-injector Program," Nucl. Instr. and Methods in Physics Res. ,A272 (1988), 222-226.

3) A.H. Lumpkin, etal., "Initial Observations of High-Charge, Low-Emittance Electron Beams at HIBAF," Proceedings of the 12. International Free-Electron Laser Conference, Paris, France, September 16-21, 1990 (to appear in J. Nucl. Instr. and Methods in Physics Res. ).

4) D.W. Feldman, etal, "Performance of the Los Alamos HIBAF Accelerator at $17 \mathrm{Mev}$, " Proceedings of the 12 th International Free-Electron Laser Conference, Paris, France, September 16-21, 1990 ( to appear in $J$. Nucl. Instr. and Methods in Physics Res. ).

5) D.W. Feldman, etal, "Experimental Results from the Los Alamos FEL Photo-injector," to be published in IEEE Journal of Quant. Electr., December 1991.

6) P.G. O'Shea, etal, "Performance or the Photoinjector Accelerator for the Los Alamos Free-Electron Laser," Proceedings of the IEEE Particle Accelerator Conference, San Francisco, Calif, May 1991.

7) J. Early, J. Barton, R. Wenzel, D. Remelius and G. Busch, "The Los Alamos FEL Photoinjector Drive Laser," to be published in IEEE J. of Quantum Electronics, December 1991.

8) A.H. Lumpkin, "The Next Generation of RF-FEL (Free-Electron Laser Diagnostics: Synchroscan and Dual-Sweep Streak Camera Techniques, " Proceedings of the 12 in International Free-Electron Laser Conference, Paris, France, September 16-21, 1990 (to appear in J. Nucl. Instr. and Methods in Physics Res.).

9) B.E. Carlsten, etal, " Accelerator Design and Calculated 
Performance of the Los Alamos HIBAF Facility," Nucl. Inst. and Methods in Physics Res.,A296 (1990),687-696.

10) A.H. Lumpkin and J.W. Early, " First Dual-Sweep Streak Camera Measurements of a Photoelectric Injector Drive Laser," This issue.

Table Caption

1. Drive laser operating specifications

\section{Figure Captions}

1. Current drive laser configuration

2. Optical fiber - multipass grating pulse compressor

3. Autocorrelator trace of compressed mode-locked pulse

4. Lamp current pulse

5. Amplifier system output

6. A typical 20 microsec wide $527 \mathrm{~nm}$ macropulse

7. KTP conversion efficiency

8. Location of diagnostic CCD cameras

9. Amplitude stabilization of the oscillator output

10. Drive laser output spatial mode

11. Electron beam profile resuiting from the imaging of the drive laser light onto the photocathode into the letters "FEL"

12. Pointing stability of the drive laser output

13. Variation in laser output mode

14. Short-term drive laser phase drift

15. Long-term drive laser phase drift 
․

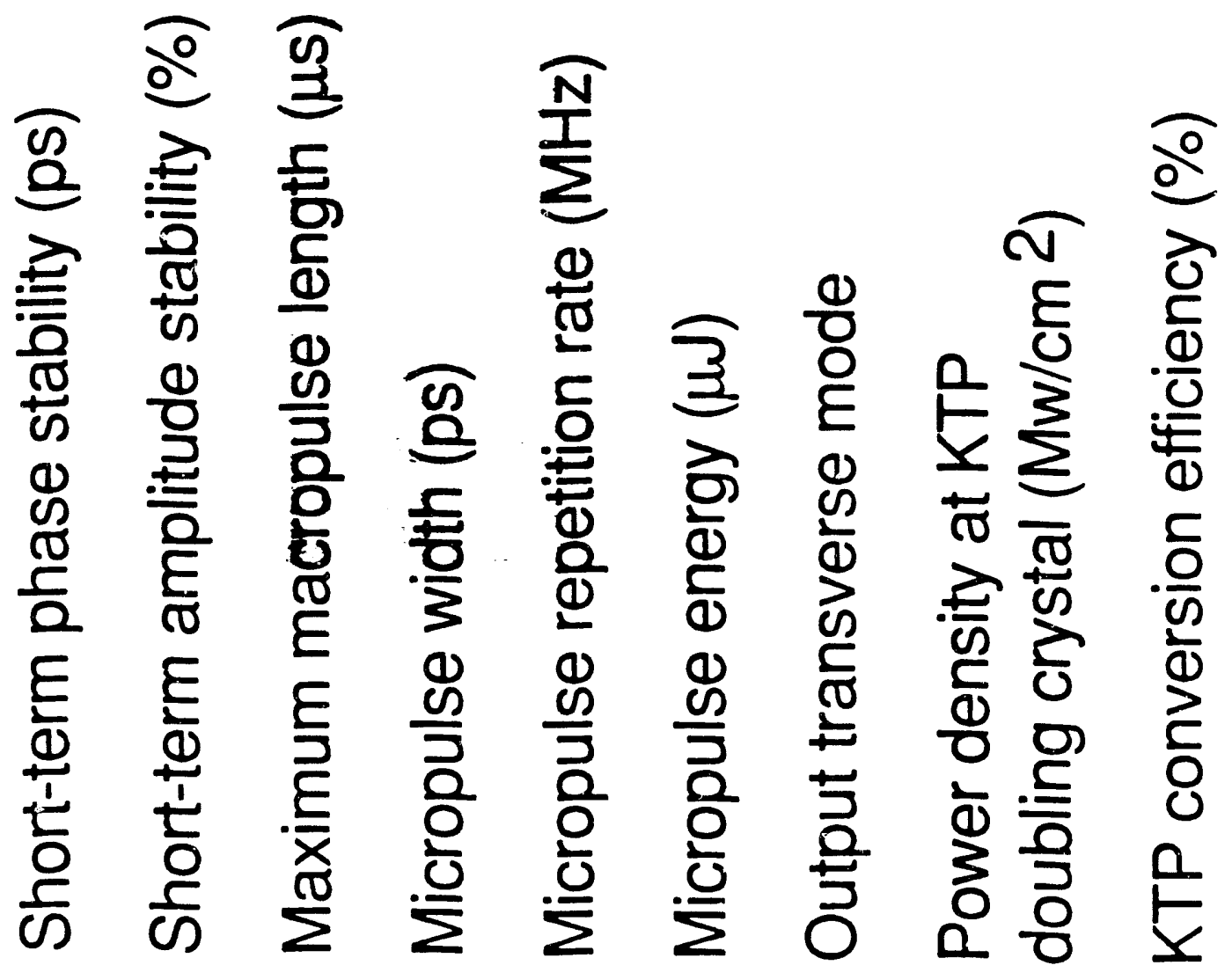




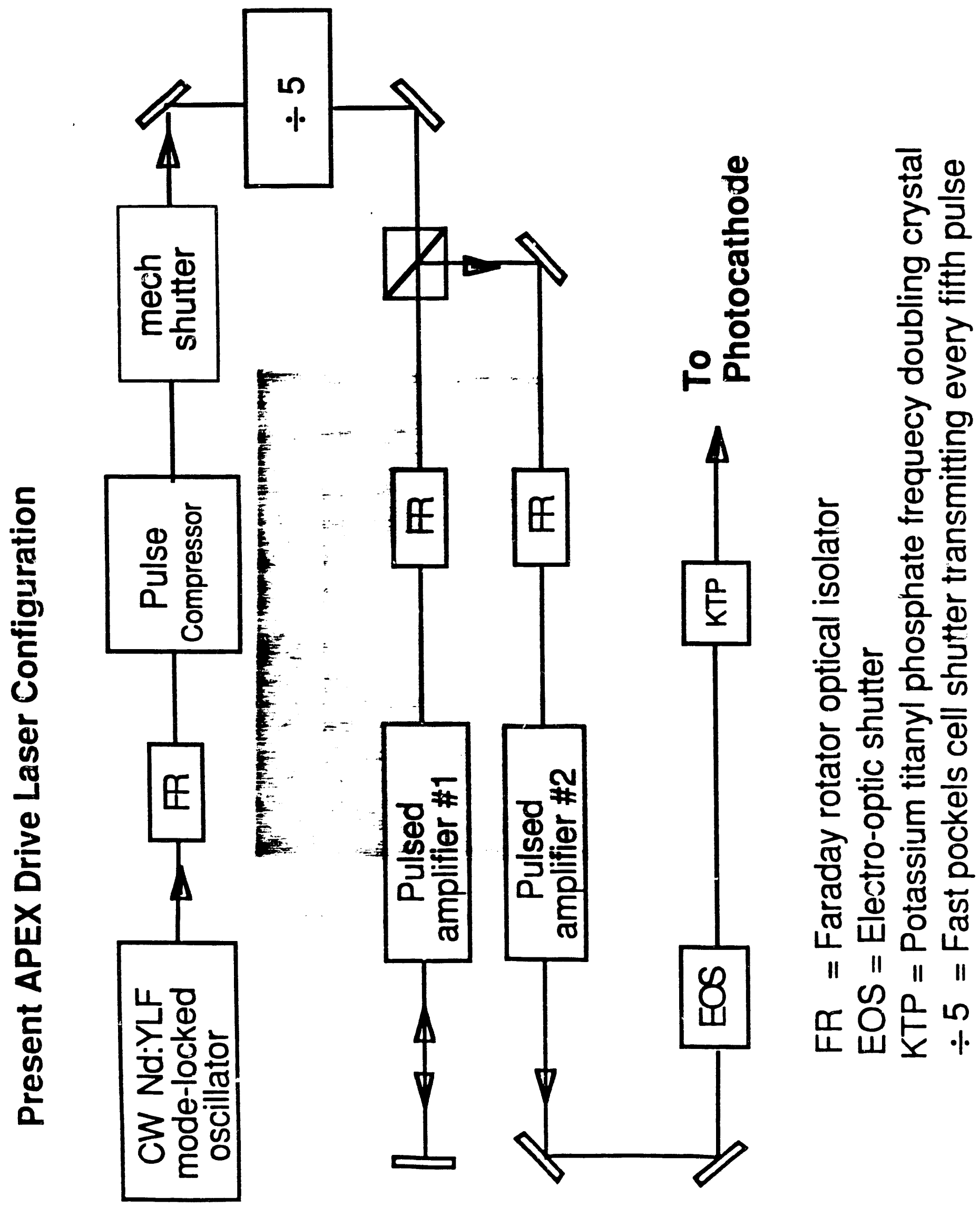




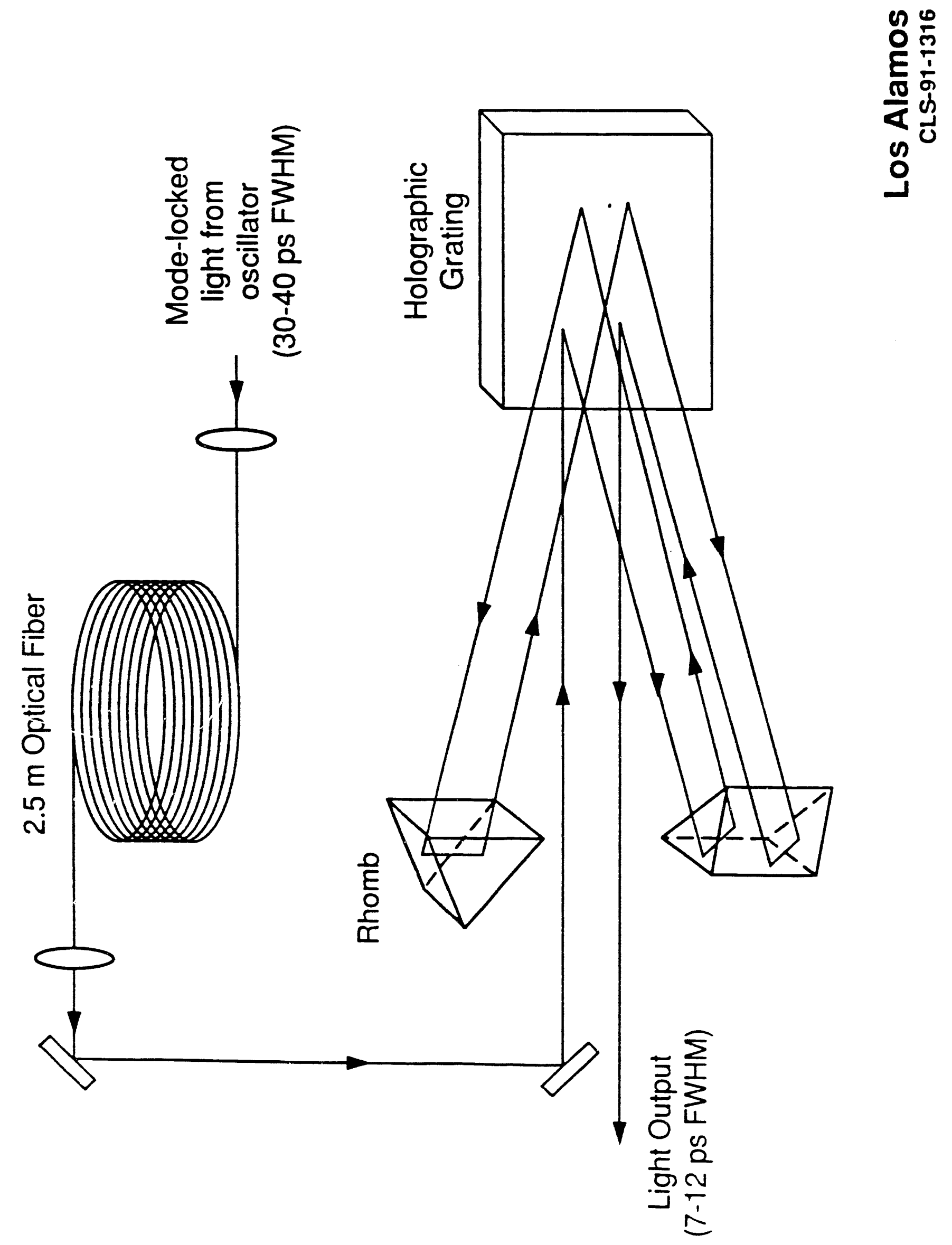




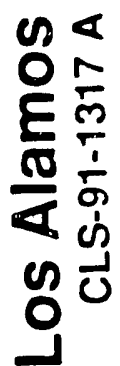

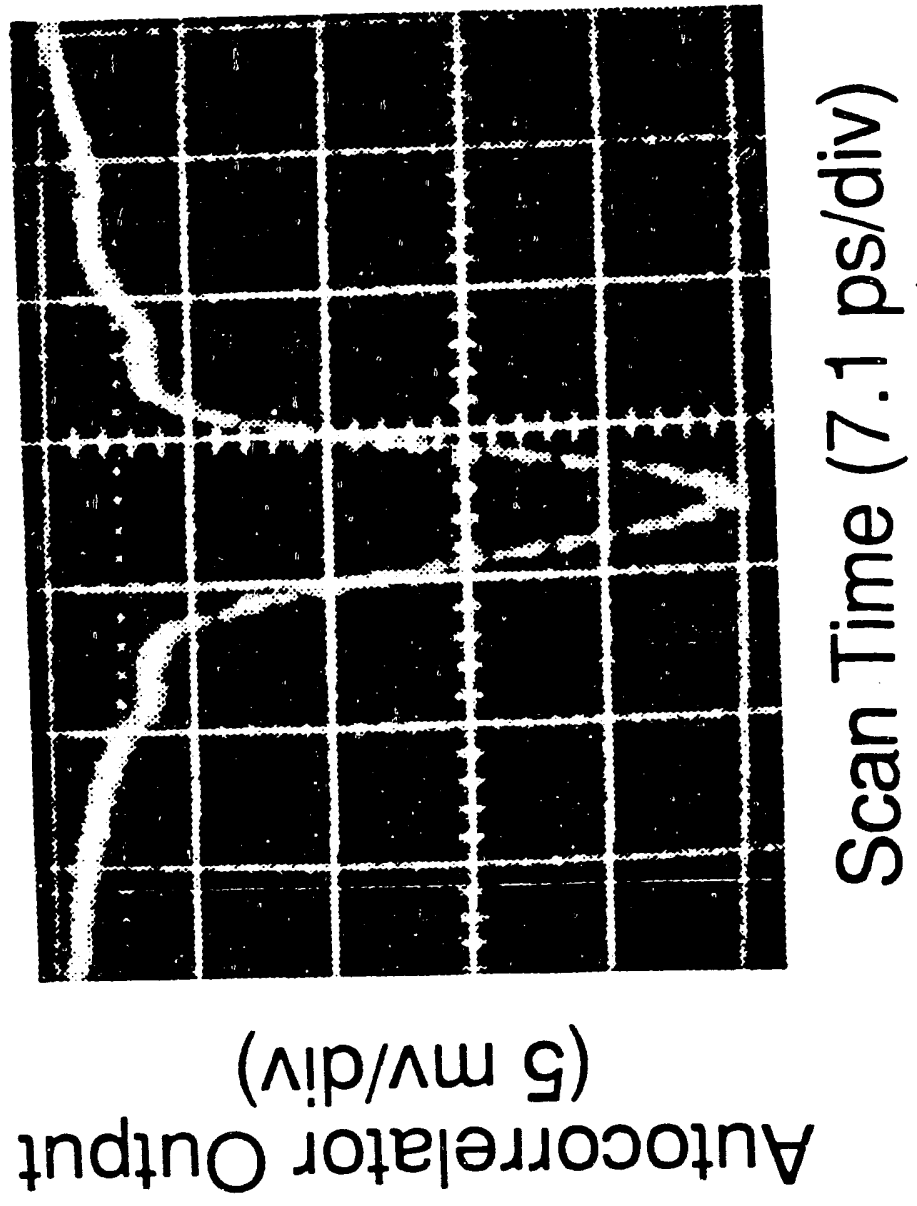




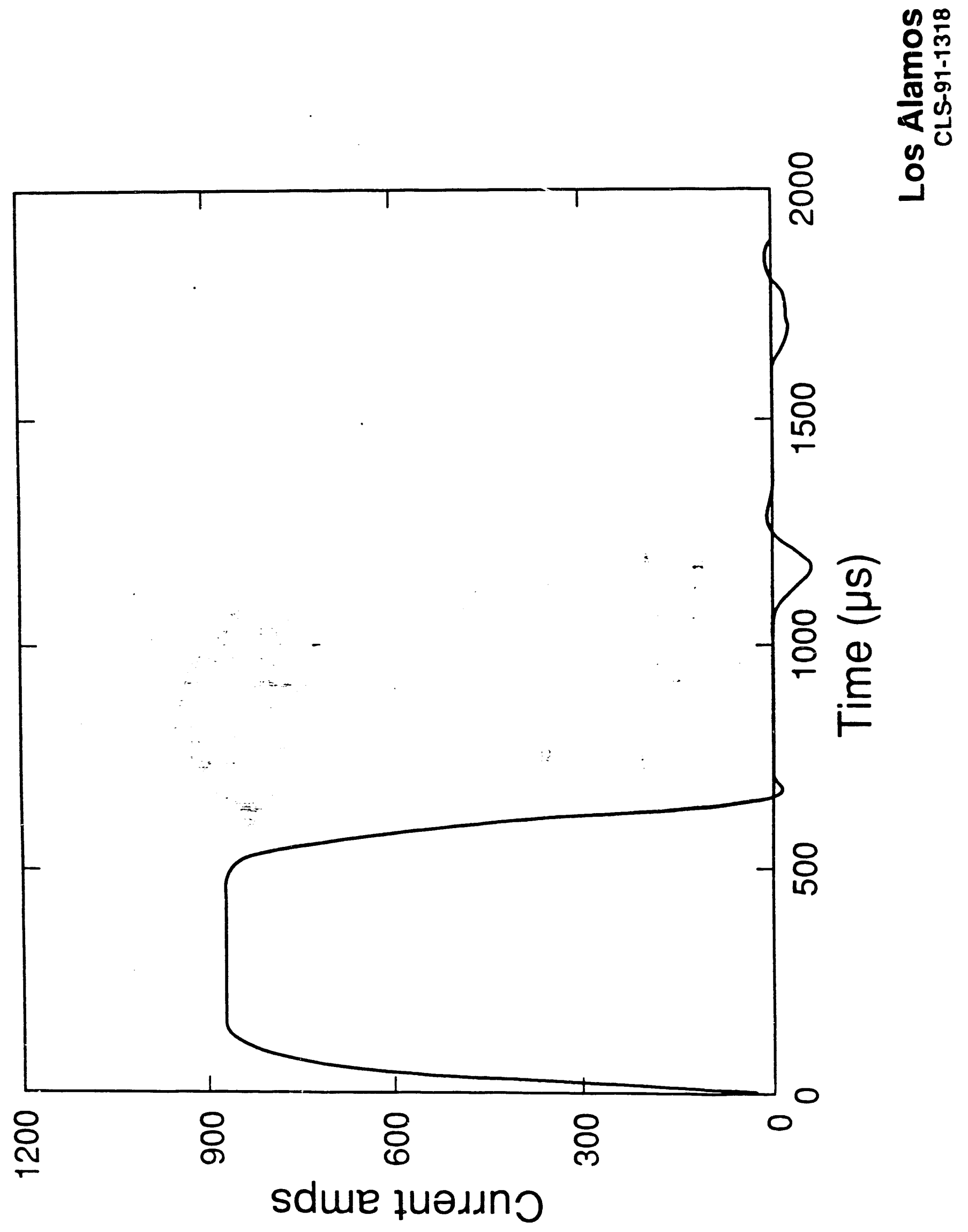


赵尔

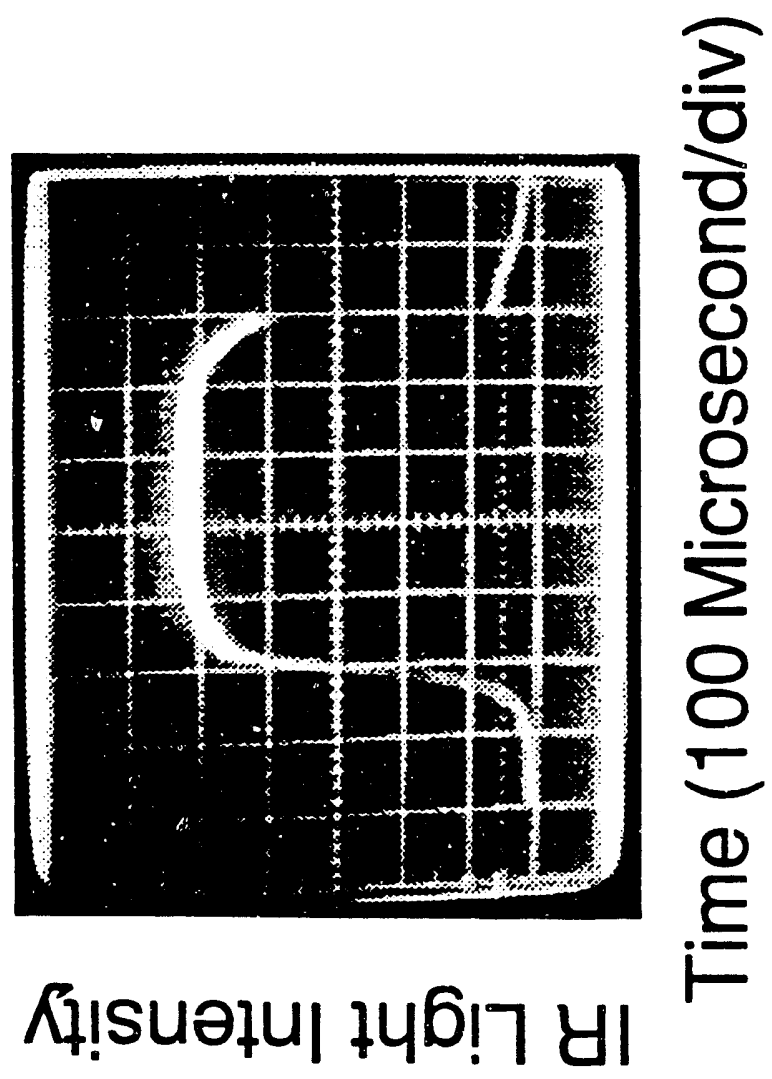



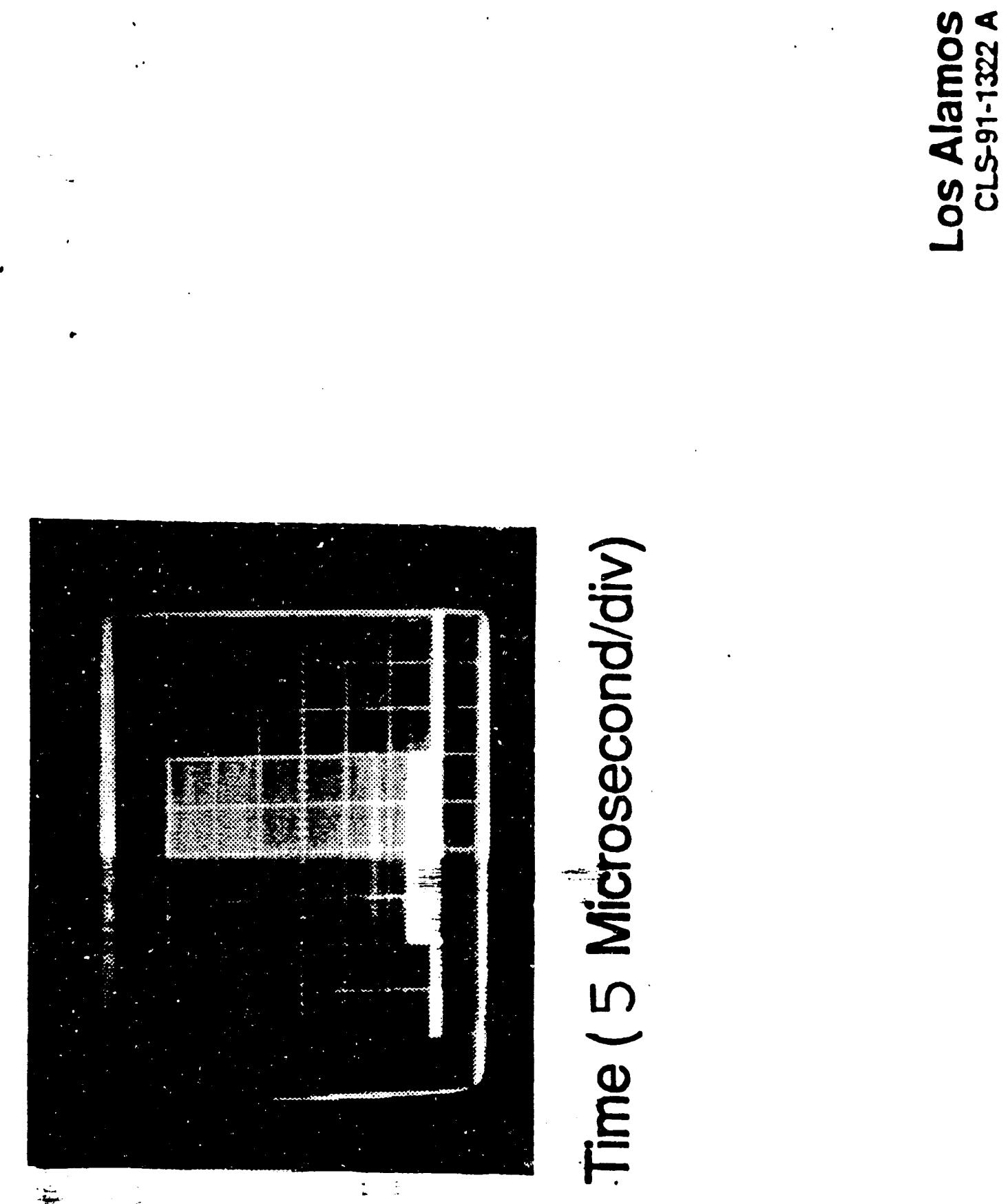

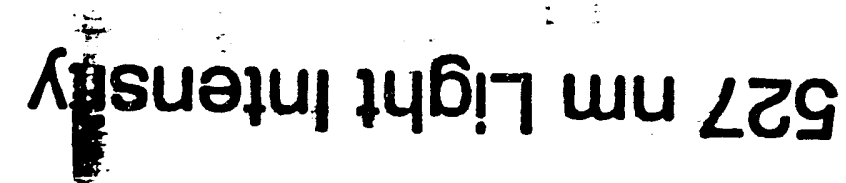

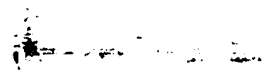




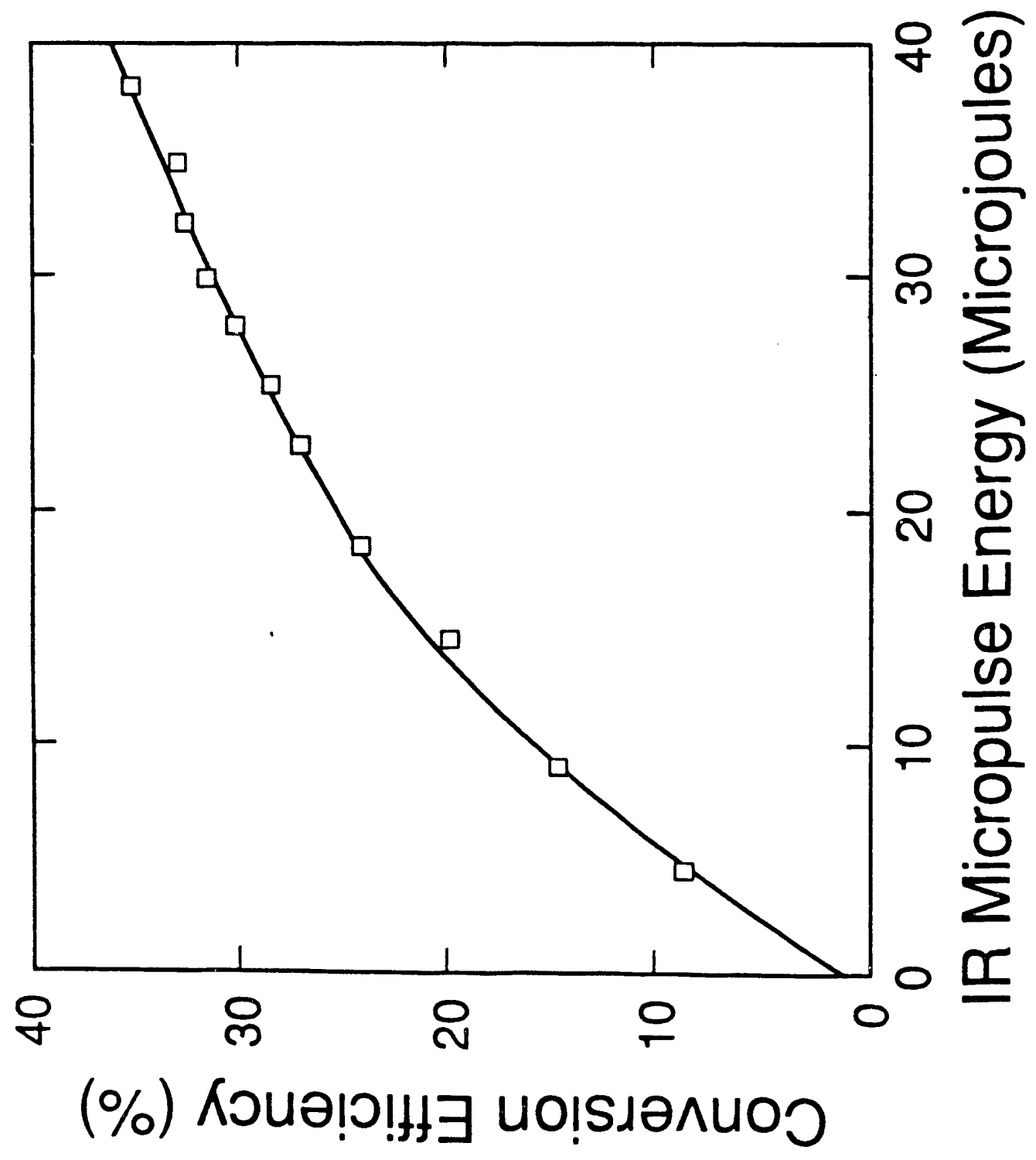




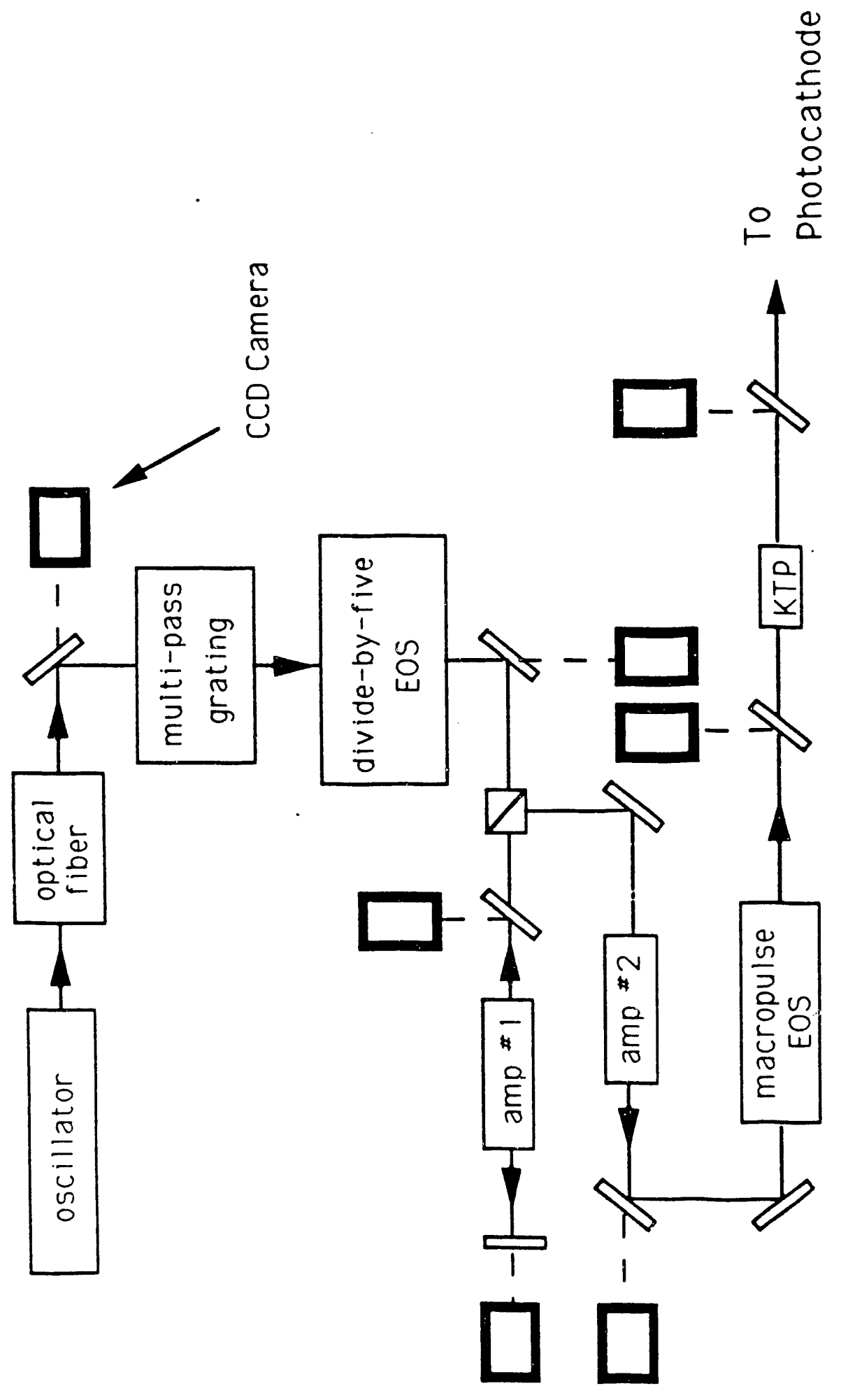




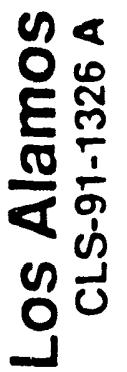
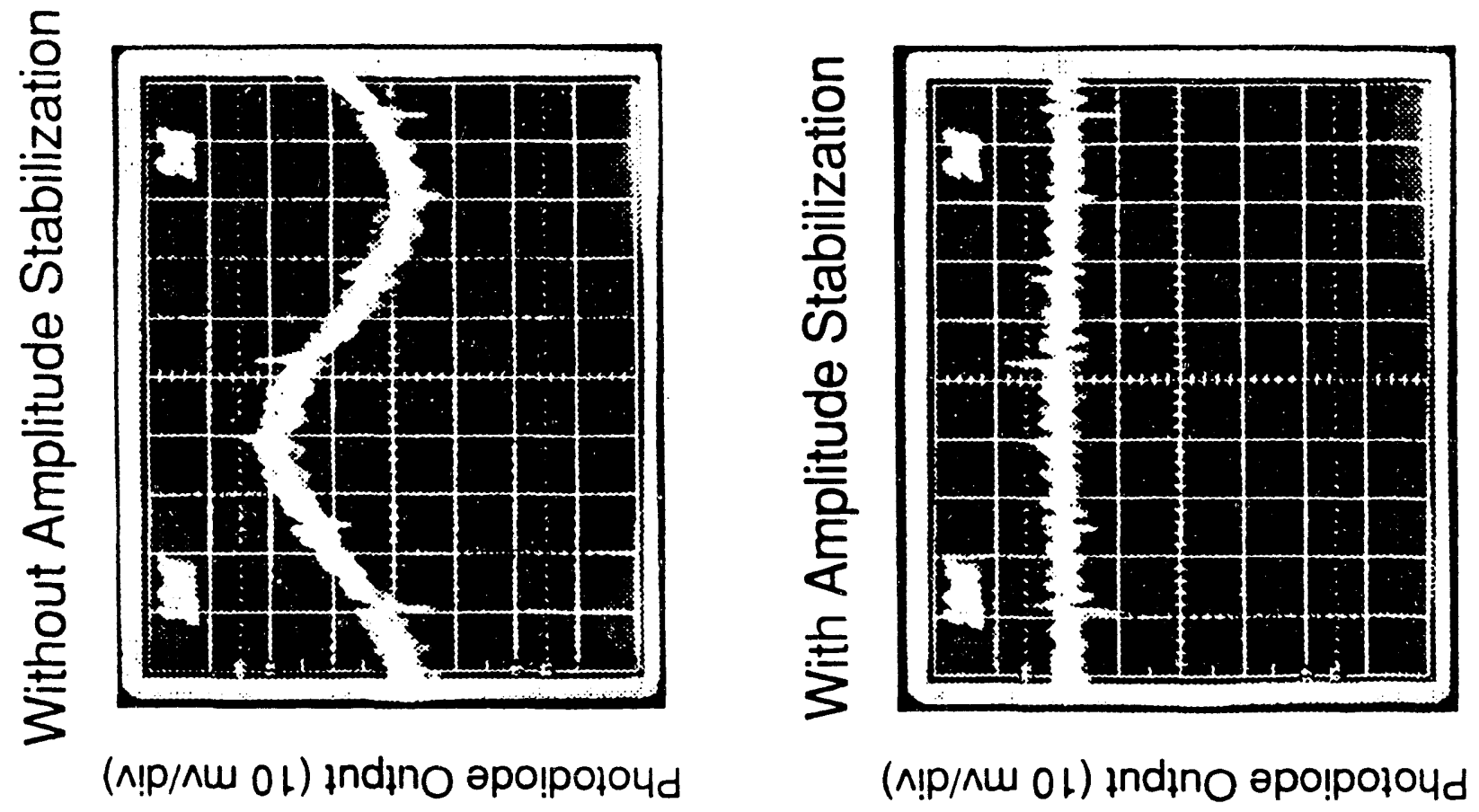


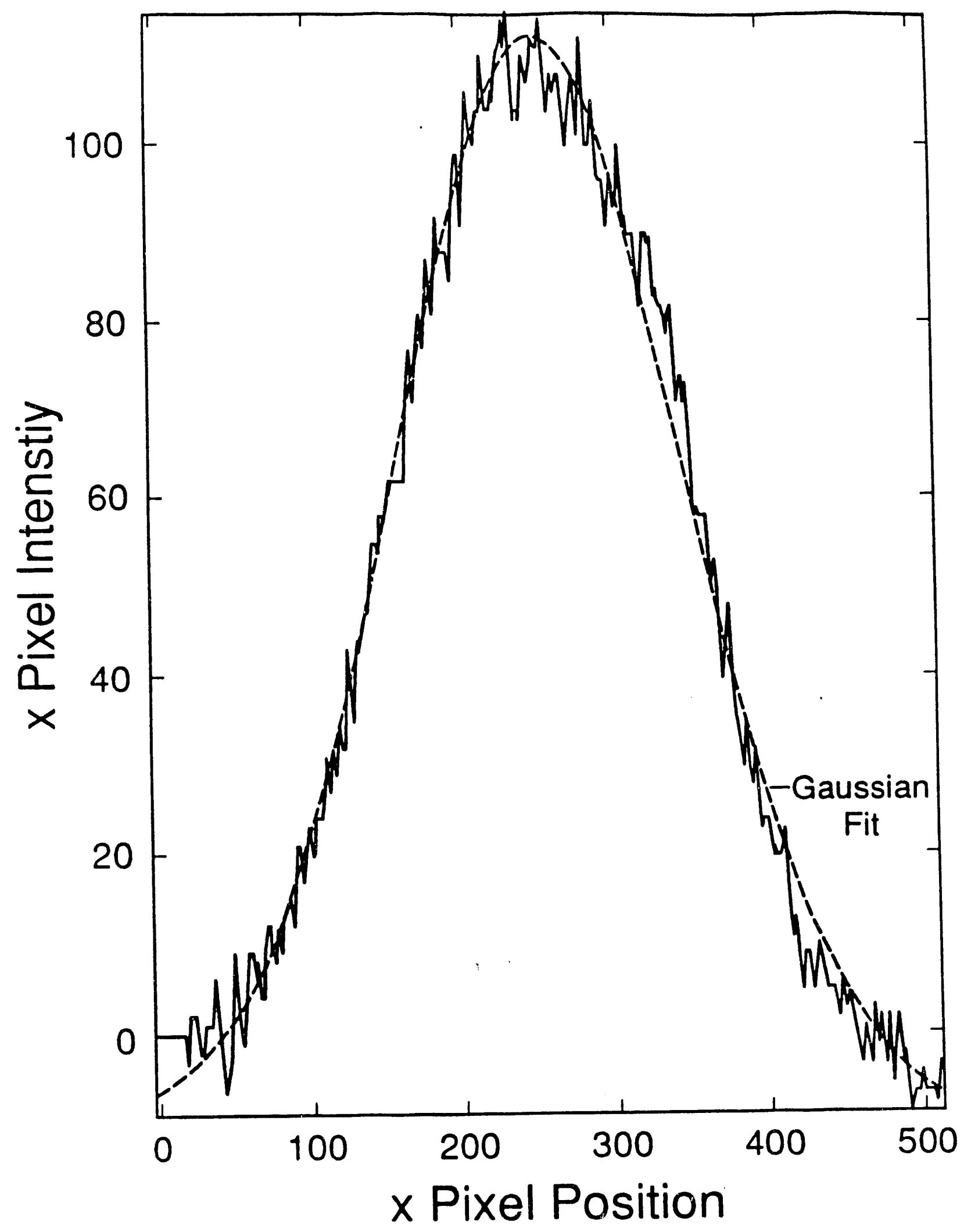

Los Alamos 



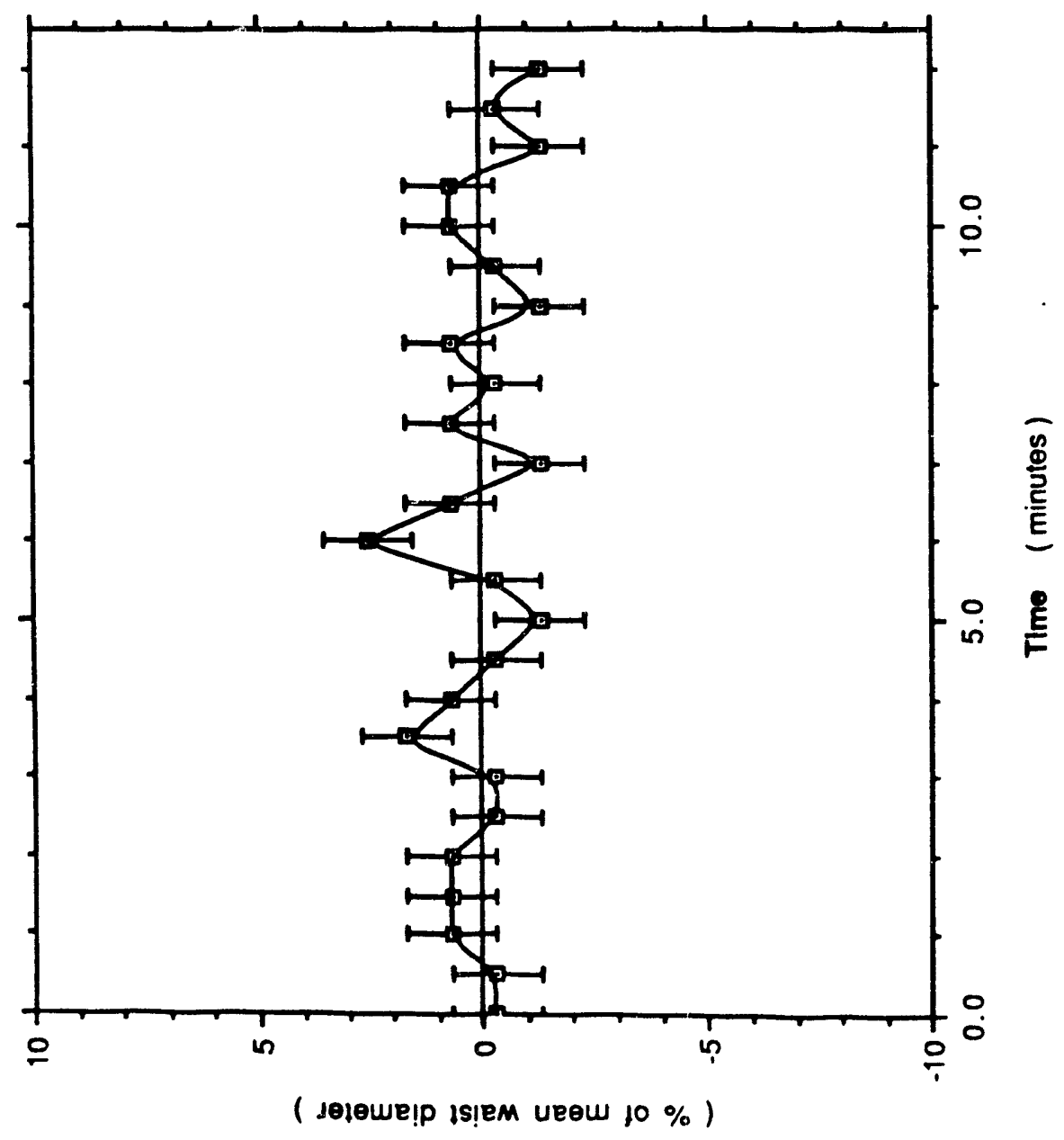

uollisod opow ul uolfepten 


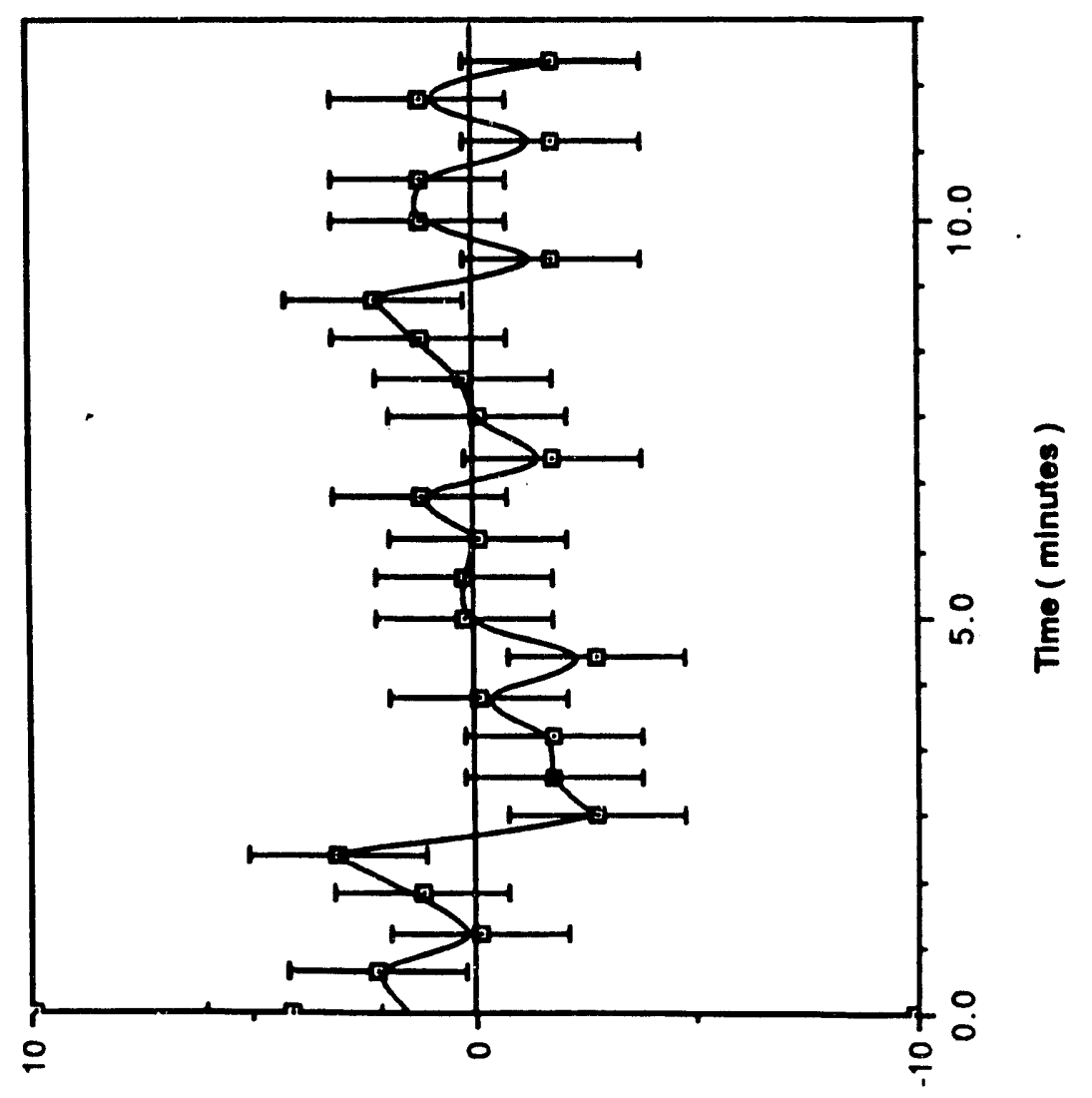

( sөlөше!p is!em ueөш jo \% )

Isiem opow ul volfejas 


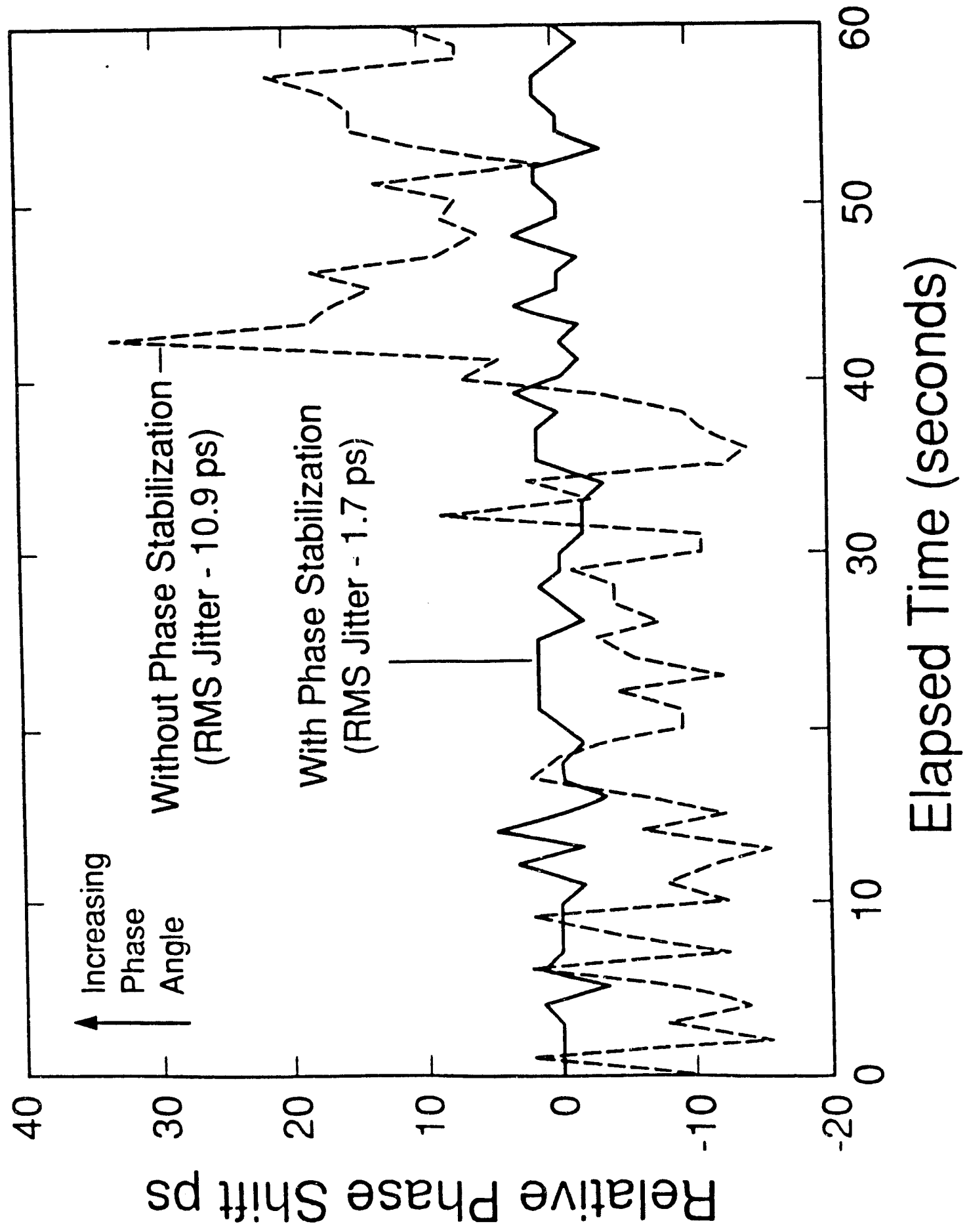




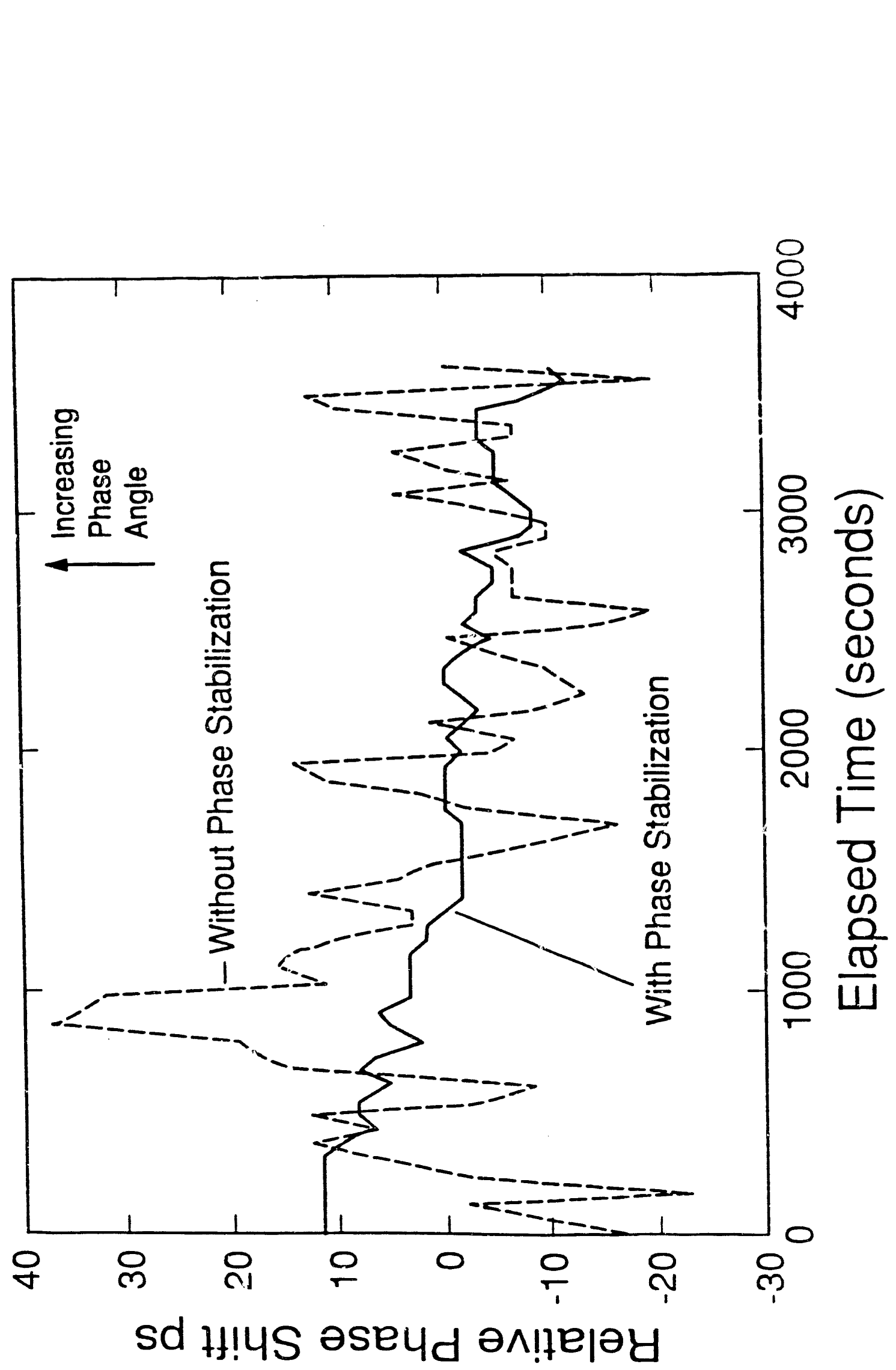

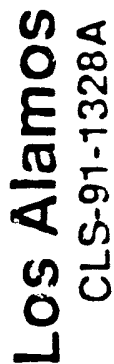



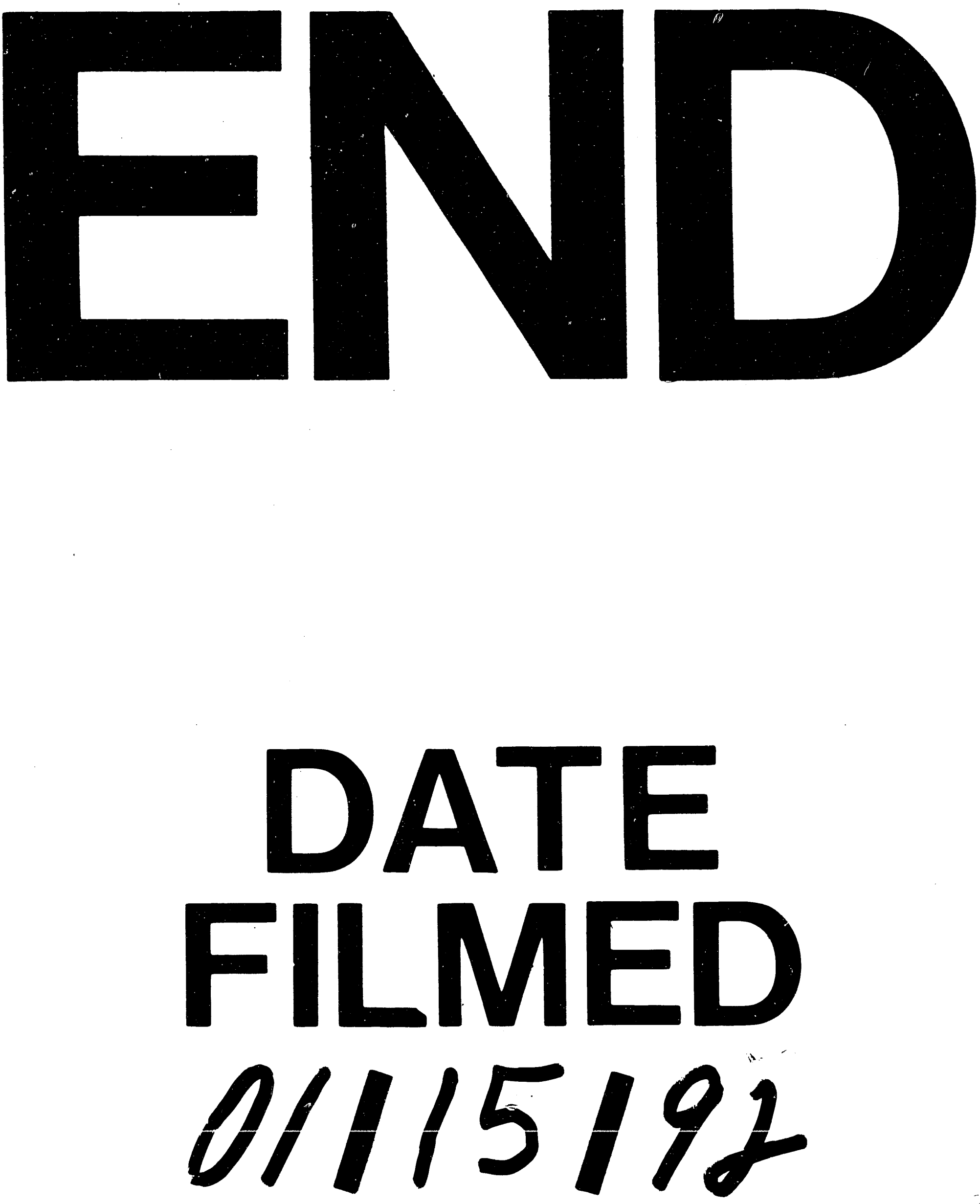
